\title{
Electronic Cigarette: are we introducing its use too early?
}

\section{Gambhir Shrestha}

\author{
School of Public Health and Community Medicine, BP Koirala Institute of Health Sciences, \\ Dharan, Nepal \\ gamvir.stha@gmail.com
}

Electronic cigarette or e-cigarette (EC) is a battery-powered electronic device that delivers an aerosol by heating a solution that users inhale. This vaporizer simulates the feeling of smoking. The main constituents of the solution are nicotine, propylene glycol, with or without glycerol and flavouring agents. ${ }^{1}$ This product was introduced as nicotine-replacement product to control tobacco smoking and as harm reduction strategy.

E-cigarettes are easily available today in the market of most of the countries. They may come in various shapes like their counter part conventional cigarettes or cigars and also in attractive forms like pens and USB memory sticks. ${ }^{1}$ Now-a-days people perceive that e-cigarettes are effective for smoking cessation especially the adolescents. ${ }^{2}$ This may be the reason for the increasing trend of e- cigarettes smoking in recent years. ${ }^{3}$ However, there is a debate whether these products might help some people mainly who are heavily addicted to tobacco smoking but there are many serious concerns regarding its safety, effectiveness and potential for their addiction. There have been very limited researches done in this perspective.

In available literatures, there are mixed results regarding safety and hazards of ECs. Some studies support the use of ECs to control tobacco smoking cessation ${ }^{4-6}$ while a systematic review reported ECs cannot be regarded as safe, even though they probably are less harmful than conventional cigarettes. ${ }^{7}$ Health effects related to its use are nicotine over dose due to ingestion, dermatological contact and respiratory irritation. Nicotine poisoning among children have been found due to nicotine cartiledge $e^{8}$ and it is advised to keep away from children, pregnant and lactating mothers. Since it has been recently introduced we are still unaware about its long- term health effects. Nicotine is the addictive component of tobacco and its presence in the solution of ECs raises the question of addiction in the long run. Second hand exposures is another concern of its use. ${ }^{9}$

Are we introducing its use very early without measuring benefits versus harm? There is inadequate evidence if the EC might help with quitting smoking or with cutting down. Have we properly addressed the dual use of EC and conventional cigarette in heavy smokers?

More researches are required to access the effectiveness of ECs in tobacco smoking cessation and harm reduction strategy over other nicotine replacement therapy and also to find out the long term health effect of its use.

\section{REFERENCES}

[1] World Health Organization. Electronic nicotine delivery systems Report by WHO. 2014.

[2] Camenga DR, Cavallo DA, Kong G, Morean M, Connell CM, Simon P, Bulmer SM, KrishnanSarin S. Adolescents' and young adults' perceptions of electronic cigarettes for smoking cessation: A focus group study. Nicotine \& Tobacco Research. 2015:ntv020.

[3] Zhu SH, Gamst A, Lee M, Cummins S, Yin L, Zoref L. The use and perception of electronic cigarettes and snus among the US population. PloS one. 2013;8(10):e79332.

[4] Bullen C, Howe C, Laugesen M, McRobbie H, Parag V, Williman J, Walker N. Electronic cigarettes for smoking cessation: a randomised controlled trial. The Lancet. 2013;382(9905):1629-37.

[5] Caponnetto P, Campagna D, Cibella F, Morjaria JB, Caruso M, Russo C, Polosa R. EffiCiency and Safety of an eLectronic cigAreTte (ECLAT) as tobacco cigarettes substitute: a prospective 12-month randomized control design study. PloS one. 2013;8(6):e66317. 


\section{Gambhir Shrestha}

[6] Polosa R, Caponnetto P, Morjaria JB, Papale G, Campagna D, Russo C. Effect of an electronic nicotine delivery device (e-Cigarette) on smoking reduction and cessation: a prospective 6month pilot study. BMC public health. 2011;11(1):1.

[7] Pisinger C, Døssing M. A systematic review of health effects of electronic cigarettes. Preventive medicine. 2014;69:248-60.

[8] Durmowicz EL. The impact of electronic cigarettes on the paediatric population. Tobacco control. 2014;23(suppl 2):ii41-6.

[9] Collaco JM, Drummond MB, McGrath-Morrow SA. Electronic cigarette use and exposure in the pediatric population. JAMA pediatrics. 2015;169(2):177-82. 